\title{
Tanshinone IIA increases protein expression levels of PERK, ATF6, IRE1 $\alpha$, CHOP, caspase-3 and caspase-12 in pancreatic cancer BxPC-3 cell-derived xenograft tumors
}

\author{
TSUNG-LANG CHIU ${ }^{1,2}$ and CHIN CHENG SU ${ }^{3-6}$ \\ ${ }^{1}$ Division of Neurooncology, Neuro-Medical Scientific Center, Buddhist Tzu-Chi General Hospital; ${ }^{2}$ College of Medicine, \\ Tzu Chi University, Hualien 97004; ${ }^{3}$ Tumor Research Center of Integrative Medicine; ${ }^{4}$ Department of Surgery; \\ ${ }^{5}$ Comprehensive Breast Cancer Center, Changhua Christian Hospital, Changhua 500-06; ${ }^{6}$ School of \\ Chinese Medicine, College of Chinese Medicine, China Medical University, Taichung 40402, Taiwan, R.O.C.
}

Received February 7, 2016; Accepted January 27, 2017

DOI: $10.3892 / \mathrm{mmr} .2017 .6359$

\begin{abstract}
Tanshinone (Tan)-IIA is a derivative of phenanthrenequinone and the main active ingredient isolated from Salviae miltiorrhizae radix (Danshen). Previous studies have demonstrated that Tan-IIA increased the protein expressions levels of protein kinase RNA-like endoplasmic reticulum kinase (PERK), activating transcription factor (ATF) 6, caspase-12 and CCAAT-enhancer-binding protein homologous protein (CHOP), to induce endoplasmic reticulum (ER) stress and apoptosis in human pancreatic cancer BxPC-3 cells. However, to the best of our knowledge, the effects of Tan-IIA on pancreatic cancer cells have not been investigated in vivo. Further studies are required to elucidate the therapeutic potential of Tan-IIA in inducing ER stress in cancer cells in vivo. The present study aimed to investigate the effects of Tan-IIA on the expression of ER stress-related proteins in BxPC-3-derived xenograft tumors. A total of 30 male severe combined immunodeficiency mice (age, 4 weeks) were implanted with BxPC-3 cells $\left(2 \times 10^{6} / 0.2 \mathrm{ml}\right)$ and subsequently treated with various doses of Tan-IIA $(0,30$ and $90 \mathrm{mg} / \mathrm{kg})$ for 4 weeks. After mice were sacrificed on day 33, the xenograft tumors were dissected and total protein was extracted for western blot analysis. The results of the present study demonstrated that Tan-IIA inhibited the growth of BxPC-3-derived xenograft tumors. In addition, Tan-IIA increased the protein expression levels of PERK, ATF6, caspase-12, inositol-requiring enzyme (IRE) $1 \alpha$, eukaryotic initiation factor (eIF) $2 \alpha$, phosphorylated (p)-c-Jun N-terminal kinase (JNK), CHOP and caspase-3 in a dose-dependent manner. These results indicated that Tan-IIA induced ER stress via increasing the protein expression levels of PERK, ATF6, caspase-12, IRE1 $\alpha$, eIF2 $\alpha$, p-JNK, CHOP and caspase-3 in BxPC-3 cells in vivo. Therefore, it may be
\end{abstract}

Correspondence to: Dr Chin Cheng Su, Tumor Research Center of Integrative Medicine, Changhua Christian Hospital, 135 Nanxiao Street, Changhua 500-06, Taiwan, R.O.C.

E-mail: succ.maeva@msa.hinet.net

Key words: tanshinone IIA, BxPC-3 cells, ER stress, in vivo hypothesized that Tan-IIA has potential for the development of novel therapeutic strategies for the treatment of patients with pancreatic cancer.

\section{Introduction}

Although many efforts have been made to improve the efficacy of therapeutic strategies for the treatment of pancreatic cancer, current chemotherapeutic options remain unsatisfactory (1). In 2016, pancreatic cancer was reported as the 4th leading cause of cancer-associated mortality in the USA, the estimated death in males was 21,450 and in females 20,330 (2), stressing the need for the development of novel therapeutic agents for pancreatic cancer. Tanshinone (Tan)-IIA $\left(\mathrm{C}_{19} \mathrm{H}_{18} \mathrm{O}_{3}\right)$ is one of the active constituents of the plant-derived traditional Chinese medicine Danshen $(3,4)$. Tan-IIA has been reported to possess anti-cancer potential; it has been demonstrated to induce apoptosis in prostate cancer cells (5). Tan-IIA has also been reported to inhibit the proliferation of A549 human non-small cell lung cancer cells via decreasing the expression of vascular endothelial growth factor and its receptor (6). Yang et al reported that Tan-IIA inhibited the growth of human glioma stem cells via inducing apoptosis in vitro and in vivo in a dose-dependent manner (7). Munagala et al suggested that Tan-IIA may have potential as a therapeutic agent for the prevention and treatment of cervical and other human papilloma virus-related types of cancer (8). It has previously been reported that Tan-IIA may exert cytotoxic effects in human pancreatic cancer MIAPaCa-2 (9) and BxPC-3 cells (10), and it induced endoplasmic reticulum (ER) stress to inhibit the growth of BxPC-3 cells in vitro (11). Further in vivo studies are required to elucidate the mechanisms underlying the ER-related effects of Tan-IIA in BxPC-3 cells. The present study investigated the in vivo effects of Tan-IIA on the expression of ER stress-related proteins in BxPC3-derived xenograft tumors.

\section{Materials and methods}

Chemicals and reagents. The BxPC-3 human pancreatic cancer cell line was obtained from the Food Industry Research and 
Development Institute (Hsinchu, Taiwan). Tan-IIA, sodium deoxycholate, leupeptin, Triton X-100, Tris-HCl, sodium pyruvate, HEPES, RPMI-1640, trypsin-EDTA, mouse anti- $\beta$-actin antibody (cat. no. A5441; Sigma-Aldrich; Merck KGaA, Darmstadt, Germany), penicillin-streptomycin, dimethyl sulfoxide, potassium phosphates and were obtained from Merck KGaA. Fetal bovine serum (FBS) and glutamine were obtained from Gibco (Thermo Fisher Scientific, Inc., Waltham, MA, USA). Tris-glycine-SDS buffer (10X), Tween-20 and glycine were obtained from Ameresco, Inc. (Framingham, MA, USA). BioMax film was obtained from Kodak (Rochester, NY, USA). Anti-protein kinase RNA-like endoplasmic reticulum kinase (PERK) (cat. no. 9956), anti-inositol-requiring enzyme $1 \alpha$

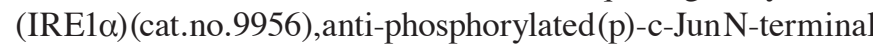
(JNK) (cat. no. 9910), anti-CCAAT-enhancer-binding protein homologous protein (CHOP) (cat. no 9956) and anti-caspase-3 (cat. no 9661) antibodies were obtained from Cell Signaling Technology, Inc. (Danvers, MA, USA). Anti-caspase 12 (cat. no. ab62484), anti-activating transcription factor 6 (ATF6) (cat. no. ab37149) and anti-eukaryotic initiation factor $2 \alpha$ (elF2 $\alpha$ ) (cat. no. ab5369) antibodies were obtained from Abcam (Cambridge, UK). Anti-B-cell lymphoma 2 (Bcl-2) antibody (cat. no. NB100-92142) was obtained from Novus Biologicals, LLC (Littleton, CO, USA).

Cell culture. Human pancreatic adenocarcinoma BxPC-3 cells were cultured as previously described (10,11). Briefly, BxPC-3 cells were maintained in RPMI-1640 medium supplemented with $10 \%$ FBS, 10,000 U/ml penicillin and $10 \mathrm{mg} / \mathrm{ml}$ streptomycin, at $37^{\circ} \mathrm{C}$ in a humidified atmosphere containing $5 \% \mathrm{CO}_{2}$.

In vivo studies. Cultured BxPC3 cells $\left(2 \times 10^{6} / 0.2 \mathrm{ml}\right)$ were implanted into 4-week old, male nude severe combined immunodeficiency (SCID) mice $(n=30)$ via subcutaneous injection over the flank area. Mice were maintained in a pathogen-free environment (Laboratory Animal Center of Tzu Chi University, Hualien, Taiwan). SCID mice implanted with BxPC-3 cells were randomly divided into 3 groups $(\mathrm{n}=10$ per group) to receive 3 different weekly doses of Tan-IIA $(0,30$ and $90 \mathrm{mg} / \mathrm{kg})$. Tan-IIA was dissolved in corn oil and administered intraperitoneally on weeks 1, 3 and 5 following xenotransplantation. Volumes of the xenograft tumors were measured every other week. Tumor volume was estimated according to the following formula: Tumor volume $\left(\mathrm{mm}^{3}\right)=\mathrm{LxW}^{2} / 2$, where $\mathrm{L}$ refers to tumor length and $\mathrm{W}$ refers to tumor width. On day 35 following xenotransplantation SCID mice were sacrificed by $\mathrm{CO}_{2}$ inhalation, the xenograft tumors were dissected and total protein was extracted from the tumors. Subsequently, protein expression levels of PERK, ATF6, caspase-12/caspase-3, IRE1 $\alpha$, eIF2 $\alpha$, p-JNK, CHOP and $\mathrm{Bcl}-2$ in the xenograft tumors were assessed using western blot analysis.

All experimental procedures were approved by the Institutional Animal Care and Use Committee of Tzu Chi University (approval no. CCH-AE-101-010).

Protein preparation. Total protein was extracted from xenograft tumors. Following dissection, tumors were homogenized and lysed in ice-cold whole cell lysis buffer containing protease inhibitors (BioVision, Inc., Milpitas, CA, USA). The

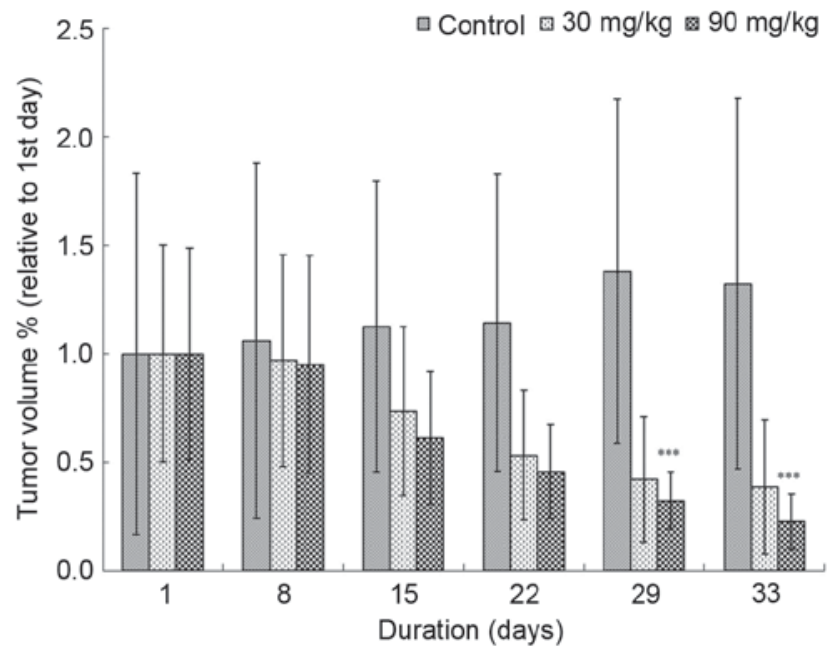

Figure 1. Effects of Tan-IIA administration on BxPC-3-derived xenograft tumors. Mice bearing BxPC-3-derived xenograft tumors were treated with various doses of Tan-IIA $(0,30$ and $90 \mathrm{mg} / \mathrm{kg})$. Tumor volumes were measured every other week. The tumor volume was estimated according to the formula: Tumor volume $\left(\mathrm{mm}^{3}\right)=\mathrm{LxW}^{2} / 2$. Tumor volume was significantly reduced following 29 days of treatment with a high dose of Tan-IIA $(90 \mathrm{mg} / \mathrm{kg})$ compared with the control group. Data are expressed as the mean \pm standard deviation. ${ }^{* * *} \mathrm{P}<0.001$. Tan, Tanshinone; L, tumor length; W, tumor width.

lysates were incubated for $30 \mathrm{~min}$ at $4^{\circ} \mathrm{C}$ with agitation and were centrifuged at 12,281 x $g$ for $10 \mathrm{~min}$. Protein concentration was measured using the bicinchoninic acid protein assay kit (Pierce; Thermo Fisher Scientific, Inc.).

Western blot analysis. Western blot analysis was conducted as previously described $(10,11)$. Briefly, equal amounts of extracted protein samples $(10 \mu \mathrm{g})$ were separated by $12 \%$ SDS-PAGE (Bio-Rad Laboratories, Inc., Hercules, CA, USA) and transferred onto polyvinylidene difluoride membranes, which were blocked for $1 \mathrm{~h}$ at $4^{\circ} \mathrm{C}$ with blocking buffer [5\% dried skimmed milk in solution containing $50 \mathrm{mM}$ Tris- $\mathrm{HCl}$ ( $\mathrm{pH} \mathrm{8.0),} 2 \mathrm{mM} \mathrm{CaCl}_{2}, 80 \mathrm{mM}$ sodium chloride, $0.05 \%$ Tween-20 and $0.02 \%$ sodium azide (Merck KGaA)]. The membranes were incubated for $2 \mathrm{~h}$ at room temperature with the following primary antibodies: PERK, ATF6, caspase-3, caspase-12, IRE1 $\alpha$, eIF2 $\alpha$, p-JNK, CHOP, Bcl-2 (all diluted to 1:1,000) and $\beta$-actin (diluted to 1:5,000). Subsequently, they were incubated at room temperature for $1 \mathrm{~h}$ with anti-rabbit (cat. no. sc-2004) or anti-mouse (cat. no. sc-2005) immunoglobulin G-horseradish peroxidase-conjugated secondary antibodies (1:5,000; Santa Cruz Biotechnology Inc., Dallas, TX, USA). The membranes were washed 3 times for 10 min with 1X PBS with $0.05 \%$ Tween-20. The protein bands were visualized on X-ray film using an enhanced chemiluminescence detection system (PerkinElmer, Inc., Waltham, MA, USA) and quantified using ImageJ version 1.44 (National Institute of Health, Bethesda, MD, USA).

Statistical analysis. The statistical significance of the difference between groups was assessed by one-way analysis of variance followed by Dunnett's test. Data are expressed as the mean \pm standard deviation. $\mathrm{P}<0.05$ was considered to indicate a statistically significant difference. The analysis was 
A
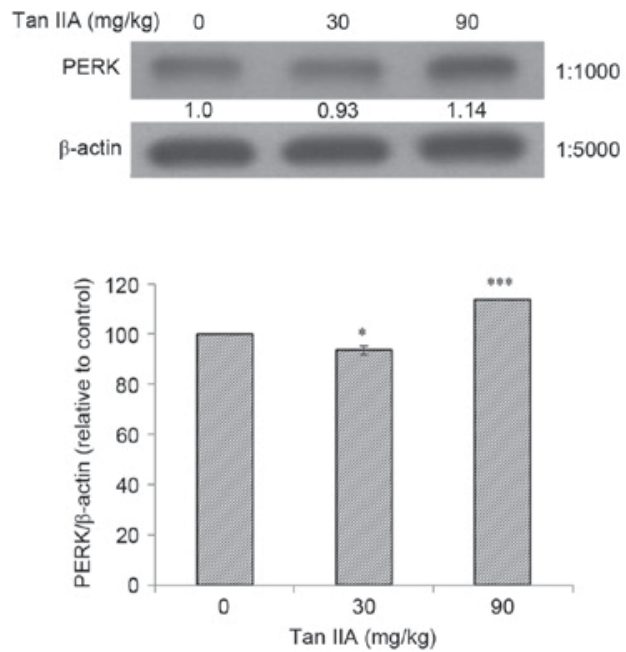

C
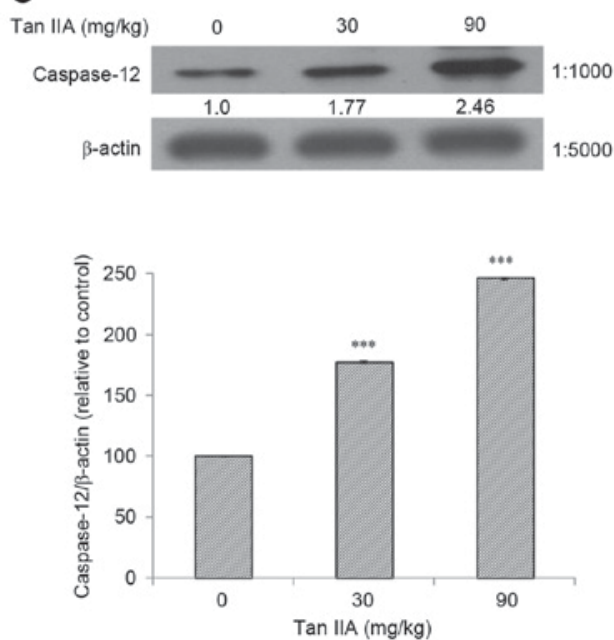

B
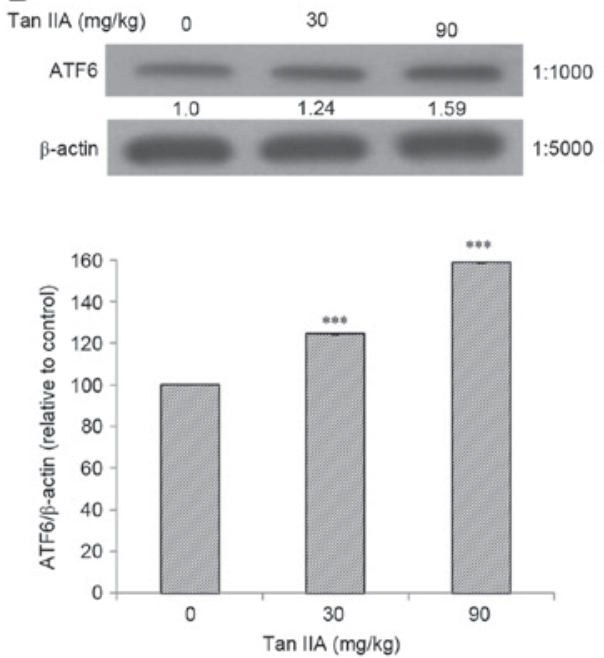

D
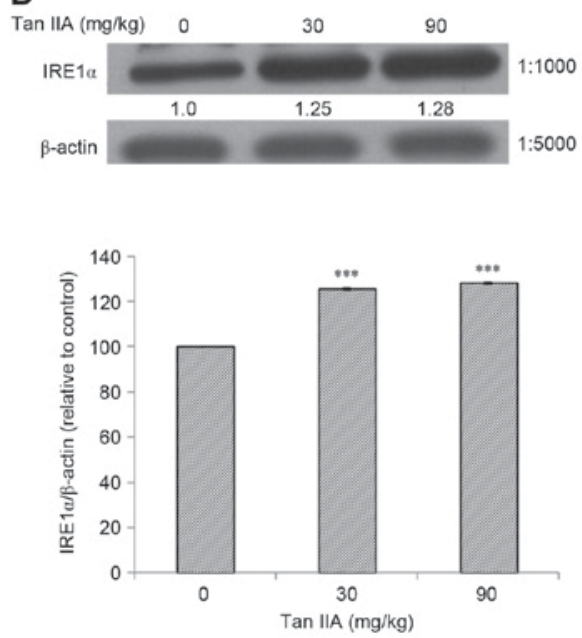

Figure 2. Protein expression levels of PERK, ATF6, caspase-12 and IRE1 $\alpha$ in BxPC-3-derived xenograft tumors following treatment with Tan-IIA. Mice bearing BxPC-3-derived xenograft tumors were treated with various doses of Tan-IIA (0, 30 and $90 \mathrm{mg} / \mathrm{kg})$. Tumors were dissected and western blot analysis was performed to assess protein expression levels. Tan-IIA increased the protein expression levels of (A) PERK, (B) ATF6, (C) caspase-12 and (D) IRE1 $\alpha$ in a dose-dependent manner. Data are expressed as the mean \pm standard deviation. ${ }^{*} \mathrm{P}<0.05,{ }^{* * * *} \mathrm{P}<0.001$ compared with the control group. PERK, protein kinase RNA-like endoplasmic reticulum kinase; ATF, activating transcription factor; IRE, inositol-requiring enzyme; Tan, Tanshinone.

performed using IBM SPSS software version 20.0 (IBM SPSS, Armonk, NY, USA).

\section{Results}

Effects of Tan-IIA on BxPC3-derived tumor xenografts. Mice implanted with BxPC-3-derived tumor xenografts were treated with 3 doses of Tan-IIA $(0,30$ and $90 \mathrm{mg} / \mathrm{kg}$ ) for 4 weeks. Tan-IIA was demonstrated to impair xenograft tumor growth in a dose-dependent manner (Fig. 1). In addition, protein expression of PERK, ATF6, caspase-3, caspase-12, IRE1 $\alpha$, eIF2 $\alpha$, p-JNK, $\mathrm{CHOP}$ and Bcl-2 was assessed using western blot analysis, with $\beta$-actin as an internal control. The present results revealed that Tan-IIA significantly increased the protein expression levels of PERK (Fig. 2A), ATF6 (Fig. 2B), caspase-12 (Fig. 2C), IRE1 $\alpha$ (Fig. 2D), elF2 $\alpha$ (Fig. 3A), p-JNK (Fig. 3B), CHOP (Fig. 3C) and caspase-3 (Fig. 4A) in a dose-dependent manner. Conversely, treatment with Tan-IIA resulted in a significant dose-dependent decrease in Bcl-2 protein expression levels (Fig. 4B).

\section{Discussion}

The induction of ER stress is one of the underlying mechanisms involved in therapeutic strategies for cancer (12). It has previously been reported that the activation of upstream elements, such as IRE1 $\alpha$ and PERK, consequently results in an increase in their downstream targets eIF2 $\alpha$, p-JNK and CHOP (13). $\mathrm{CHOP}$ has been demonstrated to inhibit the protein expression of Bcl-2. When the unfolded protein response exceeds a threshold, damaged cells become apoptotic, through a mechanism that may involve the caspase-12- and ATF6-mediated induction of the CHOP signaling pathway $(14,15)$. Pan et al reported that Tan-IIA may enhance the apoptosis of CaSki advanced cervical carcinoma cells, through the activation of intrinsic mitochondrial and ER stress-associated pathways (16). In addition, Chiu et al demonstrated that Tan-IIA inhibited the growth of human prostate cancer cells through the induction of ER stress in vitro and in vivo (17). The present study revealed that Tan-IIA suppressed the growth 
A
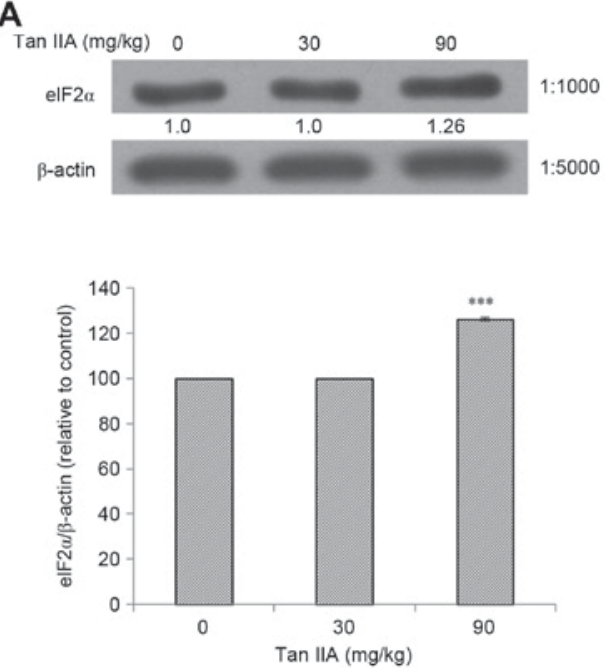

B
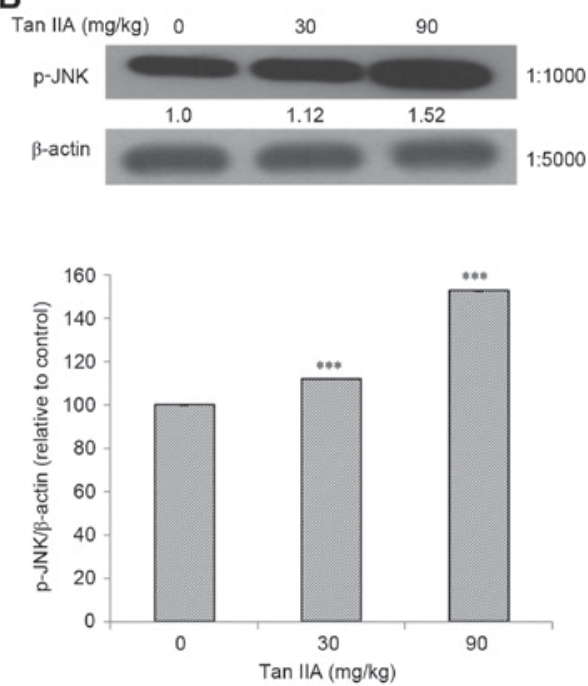

C
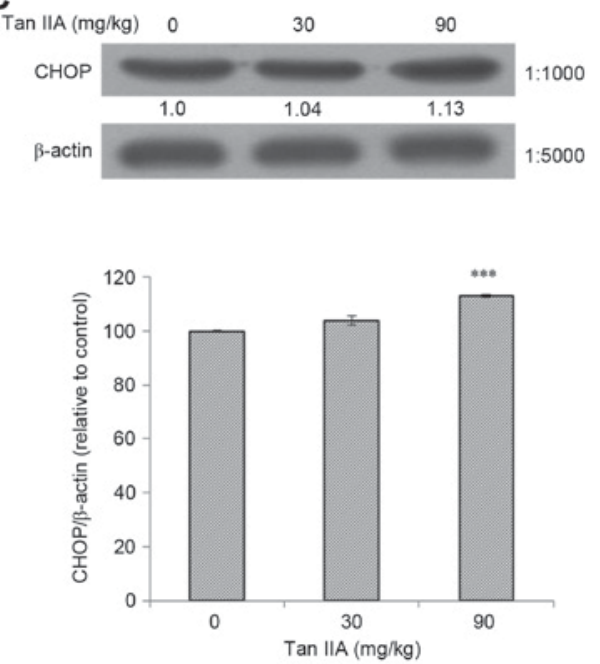

Figure 3. Protein expression levels of eIF $2 \alpha$, p-JNK and CHOP in BxPC-3-derived xenograft tumors following treatment with Tan-IIA. Mice bearing BxPC-3-derived xenograft tumors were treated with various doses of Tan-IIA $(0,30$ and $90 \mathrm{mg} / \mathrm{kg})$. Tumors were dissected and western blot analysis was performed to assess protein expression levels. Tan-IIA increased the protein expression levels of (A) eIF $2 \alpha$, (B) p-JNK and (C) CHOP in a dose-dependent manner. Data are expressed as the mean \pm standard deviation. ${ }^{* * *} \mathrm{P}<0.001$ compared with the control group. eIF, eukaryotic initiation factor; p, phosphorylated; JNK, c-Jun N-terminal kinase; CHOP, CCAAT-enhancer-binding protein homologous protein; Tan, Tanshinone.
A
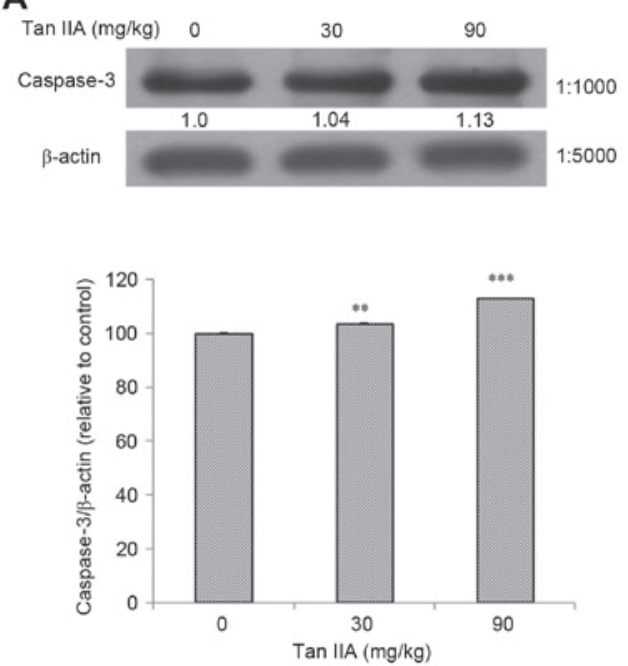

B
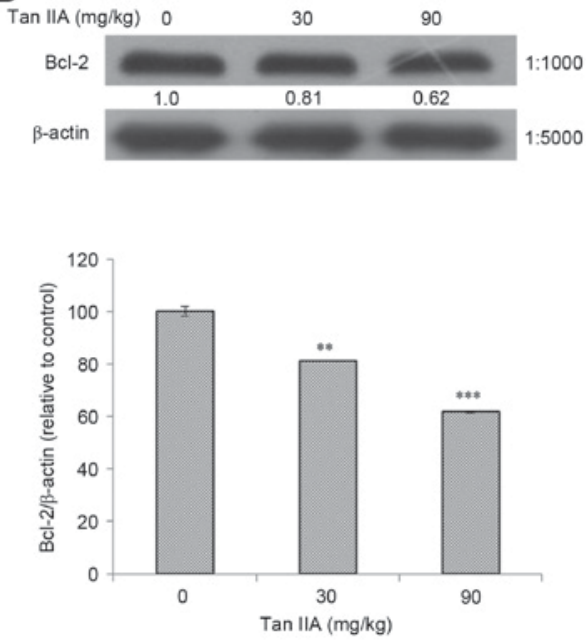

Figure 4. Protein expression levels of caspase-3 and Bcl-2 in BxPC-3-derived xenograft tumors following treatment with Tan-IIA. Mice bearing BxPC-3-derived xenograft tumors were treated with various doses of Tan-IIA $(0,30$ and $90 \mathrm{mg} / \mathrm{kg})$. Tumors were dissected and western blot analysis was performed to assess protein expression levels. Tan-IIA (A) increased the protein expression levels of caspase- 3 and (B) decreased the protein expression levels of Bcl-2 in a dose-dependent manner. Data are expressed as the mean \pm standard deviation. ${ }^{* *} \mathrm{P}<0.01,{ }^{* * *} \mathrm{P}<0.001$ compared with the control group. Bcl, B-cell lymphoma; Tan, Tanshinone.

of BxPC-3-derived xenograft tumors, as tumor volume was demonstrated to be decreased in mice following 28 days of Tan-IIA treatment compared with in untreated mice (Fig. 1). In addition, Tan-IIA increased the protein expression levels of PERK, ATF6, caspase-12, IRE1 $\alpha$, elF2 $\alpha$, p-JNK, CHOP and caspase- 3 in BxPC-3-derived xenograft tumors in a dose-dependent manner. Conversely, treatment with Tan-IIA resulted in a dose-dependent decrease of $\mathrm{Bcl}-2$ protein expression levels in BxPC-3-derived xenograft tumors. The present results indicated that Tan-IIA may promote apoptosis through the induction of ER stress in xenograft tumors derived from BxPC-3 cells. These results are in accordance with an in vitro study that demonstrated that Tan-IIA induced ER stress via increasing the expression of PERK, IRE1 $\alpha$, caspase-12 and ATF6. These proteins stimulated the overexpression of their downstream elements elF $2 \alpha$ and p-JNK, 
and the target protein CHOP, which resulted in decreased Bcl-2 expression, mitochondrial dysfunction and increased caspase-3-mediated apoptosis (11).

In conclusion, the present study suggested that Tan-IIA may exert tumor-suppressing effects via inducing ER stress in cancer cells, and may have potential as a novel therapeutic strategy for the treatment of patients with pancreatic cancer.

\section{Acknowledgements}

The present study was supported by the Research Section of the Changhua Christian Hospital, Changhua, Taiwan (grant no. 103-CCH-IRP-023).

\section{References}

1. Conroy T, Desseigne F, Ychou M, Bouché O, Guimbaud R, Bécouarn Y, Adenis A, Raoul JL, Gourgou-Bourgade S, de la Fouchardière $C$, et al: FOLFIRINOX versus gemcitabine for metastatic pancreatic cancer. N Engl J Med 364: 1817-1825, 2011.

2. Siegel RL, Miller KD and Jemal A: Cancer Statistics, 2016. Ca Cancer J Clin 66: 7-30, 2016

3. Che AJ, Zhang JY, Li CH, Chen XF, Hu ZD and Chen XG: Separation and determination of active components in Radix Salviae miltiorrhizae and its medicinal preparations by nonaqueous capillary electrophoresis. J Sep Sci 27: 569-575, 2004.

4. Zhou L, Zuo Z and Chow MS: Danshen: An overview of its chemistry, pharmacology, pharmacokinetics, and clinical use. J Clin Pharmacol 45: 1345-1359, 2005.

5. Li C, Han X, Zhang H, Wu J and Li B: The interplay between autophagy and apoptosis induced by tanshinone IIA in prostate cancer cells. Tumour Biol 37: 7667-7674, 2016.

6. Xie J, Liu J, Liu H, Liang S, Lin M, Gu Y, Liu T, Wang D, $\mathrm{Ge} \mathrm{H}$ and Mo SL: The antitumor effect of tanshinone IIA on anti-proliferation and decreasing VEGF/VEGFR2 expression on the human non-small cell lung cancer A549 cell line. Acta Pharm Sin B 5: 554-563, 2015.
7. Yang L, GuoH,Dong L, Wang L, Liu C and Wang X: Tanshinone IIA inhibits the growth, attenuates the stemness and induces the apoptosis of human glioma stem cells. Oncol Rep 32: 1303-1311, 2014.

8. Munagala R, Aqil F, Jeyabalan J and Gupta RC: Tanshinone IIA inhibits viral oncogene expression leading to apoptosis and inhibition of cervical cancer. Cancer Lett 356: 536-546, 2015.

9. Fronza M, Murillo R, Slusarczyk S, Adams M, Hamburger M, Heinzmann B, Laufer S and Merfort I: In vitro cytotoxic activity of abietane diterpenes from Peltodon longipes as well as Salvia miltiorrhiza and Salvia sahendica. Bioorg Med Chem 19: 4876-4881, 2011.

10. Huang CY, Chiu TL, Kuo SJ, Chien SY, Chen DR and Su CC: Tanshinone IIA inhibits the growth of pancreatic cancer BxPC-3 cells by decreasing protein expression of TCTP, MCL-1 and Bcl-xL. Mol Med Rep 7: 1045-1049, 2013.

11. Su CC: Tanshinone IIA could inhibit pancreatic cancer BxPC-3 cells through increasing PERK, ATF6, caspase-12 and CHOP expression to induce apoptosis. J Biomedical Sci Engineering 8: 149-159, 2015.

12. Oyadomari S and Mori M: Roles of CHOP/GADD153 in endoplasmic reticulum stress. Cell Death Differ 11: 381-389, 2004.

13. Ma Y and Hendershot LM: The role of the unfolded protein response in tumour development: Friend or foe? Nat Rev Cancer 4: 966-977, 2004.

14. Kim R, Emi M, Tanabe K and Murakami S: Role of the unfolded protein response in cell death. Apoptosis 11: 5-13, 2006.

15. Rasheva VI and Domingos PM: Cellular responses to endoplasmic reticulum stress and apoptosis. Apoptosis 14: 996-1007, 2009.

16. Pan TL, Wang PW, Hung YC, Huang CH and Rau KM: Proteomic analysis reveals tanshinone IIA enhances apoptosis of advanced cervix carcinoma CaSki cells through mitochondria intrinsic and endoplasmic reticulum stress pathways. Proteomics 13: 3411-3423, 2013.

17. Chiu SC, Huang SY, Chen SP, Su CC, Chiu TL and Pang CY: Tanshinone IIA inhibits human prostate cancer cells growth by induction of endoplasmic reticulum stress in vitro and in vivo. Prostate Cancer Prostatic Dis 2013: 315-322. 2013. 